\title{
Preliminary Investigation of Beagle Dog as Substitute for Humans in Bioequivalence Studies
}

\author{
Hari Krishan Tiwari ${ }^{{ }^{*}}$, Priya Ranjan Prasad Verma ${ }^{2}$; Tausif Monif ${ }^{1}$, Rachna Arora ${ }^{1}$ \\ and Simrit Reyar ${ }^{1}$ \\ ${ }^{1}$ Department of Clinical Pharmacology and Pharmacokinetics, Ranbaxy Research Laboratories, Plot GP-5, Sec-18, HSIIDC, \\ Old Delhi-Gurgaon Road, Gurgaon-122 015, Haryana, ${ }^{2}$ Birla Institute of Technology, Ranchi, Jharkhand, India.
}

*For correspondence: Email: hari.tiwari@ranbaxy.com; Tel: + 91-124- 4768051; Fax: +91-124-4231002

\begin{abstract}
Purpose: To assess the suitability of beagle dog as an animal model for the evaluation of formulations in bioavailability and bioequivalence studies.

Methods: A generic cetirizine $10 \mathrm{mg}$ tablet formulation was compared with another reference formulation using beagle dog as animal model. A crossover oral comparative bioavailability study was conducted on cetirizine tablet $10 \mathrm{mg}$ in healthy, male dogs under fasting conditions. The formulations were administered orally with the aid of water. Serial blood samples were collected from pre-dose to $48.0 \mathrm{~h}$ post-dose and plasma concentrations of cetirizine were determined using validated liquid chromatography-tandem mass spectrometry (LC-MS/MS) analytical method. Pharmacokinetic parameters were calculated using non-compartmental analysis while bioavailability was assessed using an analysis of variance (ANOVA) model for humans and dogs.

Results: Cetirizine plasma concentrations in dog were comparatively higher, in relation to human plasma concentrations, due to the smaller blood volume in former. There was a delay in time to reach maximum plasma concentration $\left(T_{\max }\right)$ in dog. Cetirizine formulations were found to be bioequivalent in either of the species (dog and human). The ratio (testlreference) of least-squares mean for area under plasma concentration curve from time zero to last detectable concentration $\left(A \cup C_{0-t}\right)$, area under plasma concentration curve extrapolated to infinity $\left(A \cup C_{0-\infty}\right)$ and maximum plasma concentration $\left(C_{\max }\right)$. calculated for the dogs were comparable to those for humans. $\mathrm{AUC}_{0-\mathrm{t}}, \mathrm{AUC}_{0-\infty}$ and $\mathrm{C}_{\max }$ ratios ranged within 92.81 - $106.80 \%$ for dogs and 95.43 - $104.84 \%$ for humans

Conclusion: The results suggest that beagle dogs can be used in place of humans in bioequivalence tests on generic products of cetirizine.
\end{abstract}

Keywords: Cetirizine, Beagle dog, Bioavailability, Bioequivalence, Pharmacokinetics, Noncompartmental.

Tropical Journal of Pharmaceutical Research is indexed by Science Citation Index (SciSearch), Scopus, International Pharmaceutical Abstract, Chemical Abstracts, Embase, Index Copernicus, EBSCO, African Index Medicus, JournalSeek, Journal Citation Reports/Science Edition, Directory of Open Access Journals (DOAJ), African Journal Online, Bioline International, Open-J-Gate and Pharmacy Abstracts

\section{INTRODUCTION}

Cetirizine is a selective $\mathrm{H} 1$ receptor inverse agonist used in the treatment of allergies, hay fever, angioedema, and urticaria. Generic drug companies have played a significant role in the developing world by supplying cost-effective treatment regimens [1]. To make cost effective formulation, initial development of formulations can be feasible using animal model. This can serve as guideline for planning clinical bioequivalence studies. The dog model is used frequently in formulation development and has shown utility for prediction of human bioavailability [2, 3]. During initial phase of formulation development, multiple formulations 
would require to be screened out to match with reference formulation in terms of rate and extent of absorption. The current study was planned to evaluate suitability of using beagle dog as an animal model to screen out the prototype under development closer to reference product in terms of bioavailability. This provides an additional advantage in terms of cost benefit, faster execution of study and it would also results in faster formulation development process. Kevin et al performed bioavailability studies for ritonavir formulations in which 12 out of 16 experimental ritonavir formulations in dog mirrored those obtained in human (both bioequivalent and nonbioequivalent results) [4].

Cetirizine is well-tolerated in dogs [5]. In this study, we assessed and compared the relative bioavailability of generic cetirizine $10 \mathrm{mg}$ tablet manufactured by Ranbaxy Laboratories Limited with that of innovator product Zyrtec $10 \mathrm{mg}$ tablet (containing cetirizine) manufactured by UCB Farchim SA, Switzerland.

\section{EXPERIMENTAL}

\section{Materials}

The test formulation was generic cetirizine $10 \mathrm{mg}$ tablet (batch no.1962123) manufactured by Ranbaxy Laboratories Limited, India, and reference formulation was innovator product Zyrtec $10 \mathrm{mg}$ tablet (containing cetirizine $10 \mathrm{mg}$, batch no.08C26C), manufactured by UCB Farchim SA, Switzerland. These were used for bioequivalence study in beagle dogs and humans.

\section{Subjects and criteria}

A total of 12 adult male healthy dogs aged 37 54 months with body weight ranging from 10 - 16 kg (Source: Animal Breeding and Housing Facility, Ranbaxy Research Laboratories, Gurgaon, India) were received for study. Each animal was housed in individual pen. Temperature and relative humidity were maintained at $18-29{ }^{\circ} \mathrm{C}$ and $30-70 \%$, respectively. Illumination was controlled to give approximately a sequence of $14 \mathrm{~h}$ light and $10 \mathrm{~h}$ dark. Air changes of Kennel Facility were maintained at or above 12 air changes/hour. The tablets were administered orally. Approximately $10 \mathrm{ml}$ of water was administered after dosing. Animals were divided into two groups (Group I and Group II), comprising of six animals each. Group I received cetirizine tablet $10 \mathrm{mg}$, a test formulation (T) and Group II received zyrtec 10 mg tablet, a reference formulation (R). After six days washout period, Group I administered orally with Zyrtec $10 \mathrm{mg}$ tablet and Group II with cetirizine tablet $10 \mathrm{mg}$.

Twenty four healthy, adult, male, Indian Asian human subjects aged 19-35 year were used. Body weight was ranging from $48-84 \mathrm{~kg}$. The subjects were admitted $12 \mathrm{~h}$ before dose administration in each period. The tablets were administered orally and the subjects were randomized to receive a single oral dose of test formulation or reference formulation in each period.

\section{Study design}

This trial was a single-center, two-way crossover study designed to assess the bioavailability of cetirizine $10 \mathrm{mg}$ tablet and Zyrtec $10 \mathrm{mg}$ tablet under fasting conditions in beagle dogs. Treatments were administered with approximately $10 \mathrm{~mL}$ of water and separated by adequate washout period (6 days). The dogs were fed 200 - $250 \mathrm{~g} /$ day of standard pelleted meal (Pedigree adult dog feed, Mars Pvt Ltd, India). Food was withdrawn at least $10 \mathrm{~h}$ prior to commencement of dosing and up to approximately $3 \mathrm{~h}$ post dose. Water was restricted to $1 \mathrm{~h}$ pre-dose to $2 \mathrm{~h}$ post-dose.

The study was performed as per in-house standard operating procedures based upon the guidelines [6,7], recommendations of FELASA [8], the Committee for the Purpose of Control and Supervision of Experiments on Animals (CPCSEA) guidelines for Laboratory Animal Facility [9], Guide for the Care and Use of Laboratory Animals [10]. Ethical approval was obtained from Institutional Animal Ethics Committee (IAEC protocol no 21/2010), which is monitored by CPCSEA under Ministry of Environment and Forest, Government of India.

Healthy, adult, male, human subjects aged 19-35 year were used. A bioavailability study in twenty four Asians was conducted as per the basic principles defined in US 21 CFR part 320. Ethical approval was obtained for protocol no. BE-212CETI-2008 from Sentinel Independent Ethics Committee, India. Informed consent was obtained from subjects. This study was performed as per the principles enunciated in the Declaration of Helsinki. This study was conducted by Ranbaxy Laboratories Limited, India and the data were used for comparison with dog study data. An open label, balanced, randomized, two-treatment, two-sequence, twoperiod, single-dose, cross-over design was used. A single oral dose of test formulation or reference formulation of cetirizine was administered with $240 \mathrm{~mL}$ of drinking water at ambient temperature 
after an overnight fast of at least $10 \mathrm{~h}$. Each treatment was separated by a washout period of eleven days.

\section{Bioavailability study in animals}

Twelve dogs were taken for the study and all of them completed the study. Formulations were administered through mouth (oral route). Approximately $10 \mathrm{ml}$ of water was administered after dosing. Animals were divided into two groups (Group I and Group II), comprising of six animals each. Group I received cetirizine tablet $10 \mathrm{mg}$, a test formulation ( $\mathrm{T}$ ) and Group II received zyrtec $10 \mathrm{mg}$ tablet, a reference formulation (R). After six days wash out period, Group I administered with zyrtec $10 \mathrm{mg}$ tablet and Group II with cetirizine tablet $10 \mathrm{mg}$.Blood samples approximately $0.8 \mathrm{ml}$ were collected from cephalic / saphenous vein in labeled tubes containing anticoagulant $1.8 \mathrm{mg} / \mathrm{ml}$ of EDTA (K3), predose, and at $0.167,0.500,0.833,1.000$, $1.333,1.667,2.000,2.500,3.000,4.000,6.000$, $8.000,12.000,24.000,36.000$ and $48.000 \mathrm{~h}$ post dose. Plasma (approximately $300 \mu \mathrm{ll}$ ) was separated by centrifugation at $3500 \mathrm{rpm}$ for 10 minutes at $4^{\circ} \mathrm{C}\left(+2^{\circ} \mathrm{C}\right)$ and stored in a freezer (below $-20^{\circ} \mathrm{C}$ ).

The plasma samples were analyzed by a validated method based on the Food and Drug Administration validation guidelines [11]. The plasma concentrations of cetirizine were determined using high-performance liquid chromatography fitted with mass spectrometry (LC-MS/MS) detection. Cetirizine and the internal standard (fexofenadine) were extracted from plasma using solid-phase extraction. Internal standard working solution $(50 \mu \mathrm{L})$ was added to $100 \mu \mathrm{L}$ of each plasma sample and vortexed. The samples were transferred to pre-conditioned Oasis HLB (30 mg/cc) extraction cartridges. Analyte was eluted from cartridge with $1 \mathrm{~mL}$ methanol twice. Extract was evaporated and reconstituted with $200 \mu \mathrm{L}$ mobile phase \{acetonitrile : $10 \mathrm{mM}$ ammonium formate buffer pH $3.5(90: 10 \mathrm{~V} / \mathrm{V})\}$. The extracts were injected into the LC-MS/MS system, and positive ions were monitored in multiple reaction monitoring (MRM) mode. The ion transitions $(\mathrm{m} / \mathrm{z})$ 389.4/201.3 and 502.5/466.5 were monitored for cetirizine and internal standard, respectively. Instrument setup consisted of an autosampler, an API 3000 detector and a data-processing system (Analyst 1.4.1). Linearity of cetirizine was assessed by plotting area ratios versus standard concentrations and using a linear regression weighted $1 /$ cetirizine concentration. Validated analytical range for cetirizine in plasma was 4.94 - $811.99 \mathrm{ng} / \mathrm{mL}$. Inter batch precision (CV \%) was $\leq 9.9 \%$ and accuracy (\% theoretical) ranged between 95.8 and $106.2 \%$ at low, medium and high quality control levels.

\section{Bioavailability study in human}

Twenty-two human volunteers completed the study. Two human subjects were withdrawn from study. A single oral dose of test formulation (Cetirizine Hydrochloride $10 \mathrm{mg}$ tablets) or reference formulation (Zyrtec ${ }^{\circledR} 10 \mathrm{mg}$ tablets) was administered with $240 \mathrm{~mL}$ of drinking water after an overnight fast of at least $10 \mathrm{hrs}$. Subjects received alternate treatment in the subsequent period in such a way that each subject received both the treatments at the end of the study.

Blood samples were collected at pre-dose and at $0.167,0.333,0.500,0.667,0.833,1.000$, $1.250,1.500,1.750,2.000,2.500,3.000,4.000$, $5.000,6.000,8.000,10.000,12.000,16.000$, $20.000,24.000,35.000$ and $47.000 \mathrm{~h}$ post-dose in each period. High performance liquid chromatography using mass spectrometric detection method was used to determine cetirizine content in human plasma.

Human plasma samples were analyzed by a validated method based on the Food and Drug Administration validation guideline [11]. The plasma concentrations of cetirizine were determined using high-performance liquid chromatography fitted with mass spectrometry (LC-MS/MS) detection. Cetirizine and the internal standard (diclofenac) were extracted from plasma using solid-phase extraction. Internal standard working solution $(50 \mu \mathrm{L})$ was added to $200 \mu \mathrm{L}$ of each plasma sample and vortexed. Samples were transferred to pre-conditioned HLB (30 mg/cc) extraction cartridges. The analyte was eluted from the cartridge with $1 \mathrm{~mL}$ methanol followed by $1 \mathrm{~mL}$ of water twice. The extract was evaporated and reconstituted with $500 \mu \mathrm{L}$ mobile phase \{methanol : $2 \mathrm{mM}$ ammonium acetate solution : acetic acid $(80: 20: 0.02 \mathrm{~V} / \mathrm{V} / \mathrm{V})\}$. The extracts were injected into the LC-MS/MS system, and positive ions were monitored in multiple reaction monitoring (MRM) mode. The ion transitions $(\mathrm{m} / \mathrm{z})$ $389.1 / 201.0$ and 296.0/250.0 were monitored for cetirizine and internal standard, respectively. Instrument setup consisted of an autosampler, an API 3000 detector and a data-processing system (Analyst 1.4.1). The linearity of cetirizine was assessed by plotting area ratios versus standard concentrations and using a linear regression weighted $1 /$ cetirizine $^{2}$ concentration. Validated analytical range for cetirizine in plasma was $5.00-697.21 \mathrm{ng} / \mathrm{mL}$. Inter batch precision 
(CV \%) was $\leq 9.0 \%$ and accuracy (\% theoretical) ranged between 94.4 and $98.8 \%$ at low, medium and high quality control levels.

\section{Pharmacokinetics analysis}

Non-compartmental analysis for deriving pharmacokinetic parameters was performed with WinNonlin version 5.0.1 (PharSight Corporation, Mountain View, California) on both the species concentration data. Actual time of sample collection was used for pharmacokinetic analysis of cetirizine. AUC from time 0 to the time for the last measurable concentration $\left(\mathrm{AUC}_{0-\mathrm{t}}\right)$ was calculated by linear trapezoidal method. AUC from time 0 to infinity $\left(A \cup C_{0-\infty}\right)$ was calculated as the sum of $\mathrm{AUC}_{0-\mathrm{t}}$ and ratio of last measurable plasma concentration to elimination rate constant. $\mathrm{C}_{\max }$ was calculated as the maximum measured plasma concentration over the time span specified. $T_{\max }$ was calculated as time of maximum measured plasma concentration.

\section{Statistical analysis}

Statistical analysis was performed using Statistical Analysis System software (SAS) version 9.1. Analysis of variance (ANOVA) was performed on the log natural (In)-transformed pharmacokinetic parameters $C_{\max }, A_{U} C_{0-t}$ and $\mathrm{AUC}_{0-\infty}$ using Type III sum of squares, with the main effects of formulation, period, sequence and animal /subject nested within sequence. Sequence effect, if any, was tested at the $10 \%$ level of significance using the animal / subject nested within sequence mean square as error term. Formulation and period effects were tested at $5 \%$ level of significance against the residual error (mean square error) from the ANOVA model as error term. Each ANOVA included calculation of least-squares means (LSMs), the difference between the adjusted formulation means and the standard error associated with the difference.

Ratio of test $(T)$ and reference $(R)$ formulation least square means were calculated for logtransformed pharmacokinetic parameters $\left(\mathrm{AUC}_{0}\right.$ t, $\mathrm{AUC}_{0-\infty}$ and $\left.\mathrm{C}_{\max }\right)$. It was expressed as a LSM percentage of the reference formulation. $90 \%$ confidence intervals for the ratio of LSMs were also calculated for cetirizine using two one sided hypothesis at $5 \%$ level of significance.

Test and reference formulation ratio for $\mathrm{C}_{\max }$, $\mathrm{AUC}_{0-\mathrm{t}}$ and $\mathrm{AUC}_{0-\infty}$ for both species were compared statistically using the parametric t-test at $5 \%$ level of significance. Formulations were considered bioequivalent if $90 \%$ confidence intervals (Cls) and ratios were within the regulatory acceptance range of 80.00 to 125.00 $\%$.

\section{RESULTS}

\section{Pharmacokinetics and statistics}

For the beagle dogs, the arithmetic mean \pm (SD) values for $T_{\max }, C_{\max } A \cup C_{0-t}$ and $A \cup C_{0-\infty}$ for test $(T)$ and reference $(R)$ formulations, respectively, were as follows: $1.8(0.67) \mathrm{h}$ and $1.9(0.79) \mathrm{h}$; 4037.68 (527.94) and 4069.04 (621.43) ng/mL; 54532.64 (15110.48) and 55492.52 (15497.44) ng.h/mL; 61831.85 (18569.05) and 62473.60 (19254.01) ng.h/mL in dog study. The AUC extrapolated observed was $<20 \%$ in all cases expect one, which implies that the sampling scheme was adequate for characterization of pharmacokinetic profile.

Point values of test/reference mean ratio for $\mathrm{C}_{\max }$, $\mathrm{AUC}_{0-\mathrm{t}}$ and $\mathrm{AUC}_{0-\infty}$ were 99.56, 98.20 and 99.17 $\%$ in the dog study. The $90 \%$ Cls of ratios were within the acceptance limits of $80-125 \%$ for cetirizine dog study. Least square mean values, ratios and $90 \%$ Cls for pharmacokinetic variables obtained from this study are summarized in Table 1.

Table 1: Least square mean values, ratios and $90 \%$ Cls for pharmacokinetic variables of cetirizine formulations in dogs

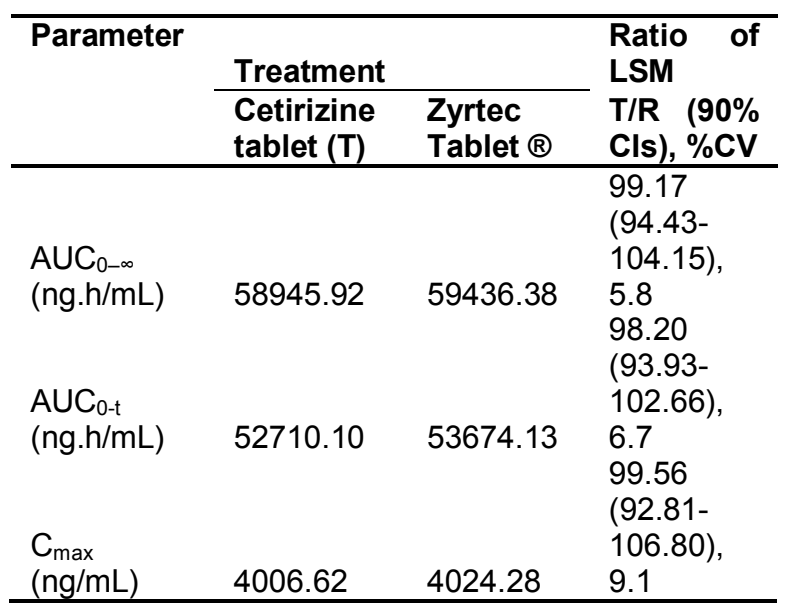

Mean dog plasma cetirizine concentrations are represented in Figure 1. This showed that both formulations exhibit identical pharmacokinetic profile and it indicates that both formulations are similar in terms of rate and extent of absorption.

Pharmacokinetic and statistical analyses were performed on data from 22 human subjects for cetirizine. Pharmacokinetic parameters were calculated and thereafter these were subjected to statistical analysis. The arithmetic mean (SD) values for $T_{\max }, C_{\max } A U C_{0-t}$ and $A U C_{0-\infty}$ for 


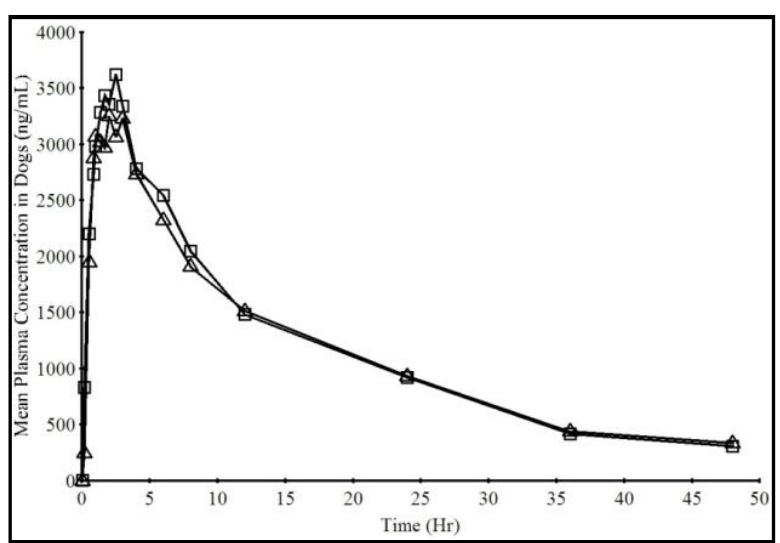

Figure 1: Linear plot of mean dog plasma cetirizine concentration $(\mathrm{ng} / \mathrm{mL})$ versus time $(h)$ of test $(\square)$ and reference $(\Delta)$ formulations

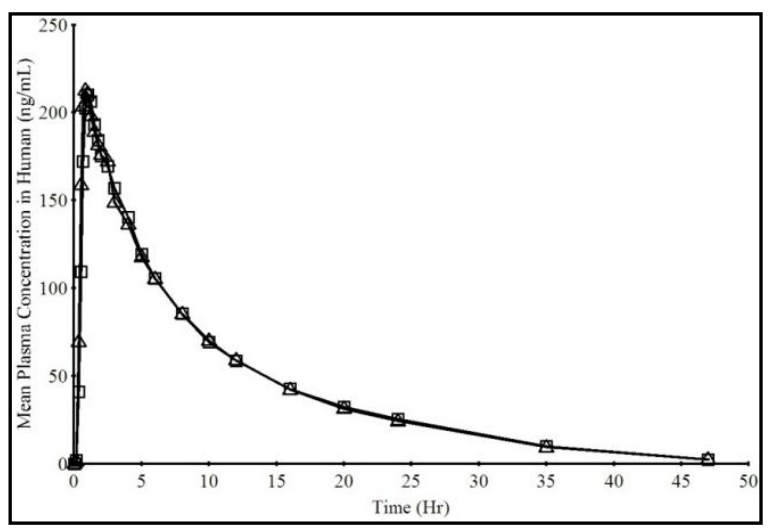

Figure 2: Linear plot of mean human plasma cetirizine concentration $(\mathrm{ng} / \mathrm{mL})$ versus time $(h)$ of test $(\square)$ and reference $(\Delta)$ formulations

cetirizine and zyrtec formulations, respectively, were as follows: $1.1(0.41)$ and $0.9(0.43) \mathrm{h}, 230.9$ (52.53) and $228.71(42.17) \mathrm{ng} / \mathrm{mL}, 2016.29$ (515.37) and 2018.09 (522.42) ng.h/mL; 2140.26 (538.20) and 2143.68 (537.93) ng.h/mL in human study. AUC\% extrap observed was $<20 \%$ in all cases for cetirizine, which implied that sampling scheme, was adequate for characterization of pharmacokinetic profile. The ratios of test $(T)$ and reference $(R)$ formulations $L S M s$ for $C_{\max }, A U C_{0-t}$ and $A_{U C} C_{0-\infty}$ were $100.02,99.99$ and $99.82 \%$ in human study. The $90 \%$ Cls for ratios were within the acceptance limits of $80-125 \%$ for cetirizine human study. Least square mean values, ratios and $90 \%$ Cls for pharmacokinetic variables obtained from human study are summarized in Table 2.

Mean human plasma cetirizine concentrations are represented in Figure 2. This showed that both formulations exhibit identical pharmacokinetic profile and it indicates that both formulations are similar in terms of rate and extent of absorption.

A parametric t-test was performed using SAS software at $5 \%$ level of significance (With PROC T-TEST) on T/R ratio of $\mathrm{C}_{\max }, \mathrm{AUC}_{0-\mathrm{t}}$ and $\mathrm{AUC}_{0-\infty}$ of both the species (dog and human) and no statistically significant difference was observed. Table 3 represents the $p$-value observed for pharmacokinetic parameters with equal and unequal variance assumption. A $p$ value $>0.05$ indicates that no significant difference between the species.

\section{DISCUSSION}

The higher $\mathrm{C}_{\max }$ in dogs compared to humans is more likely due to smaller blood volume in dogs. Certain other factors might be accounted like high destructive mechanical forces in dogs [12], different enzyme content of gut. Further, cetirizine is a BCS class I molecule with high solubility and high permeability. $\mathrm{T}_{\max }$ was 1.8 (0.67) and $1.9(0.79) \mathrm{h}$ for test and reference formulations in dog. $T_{\max }$ was $1.1(0.41)$ and 0.9 (0.43) $\mathrm{h}$ for test and reference formulations in human, respectively. It shows delayed $T_{\max }$ in dog. This shows that cetirizine was absorbed with a $\mathrm{T}_{\max }$ around $2 \mathrm{~h}$ in dog as compared to human which was around $1 \mathrm{~h}$.

Based on the ANOVA results from dog, no significant formulation, period and sequence effect was observed for log transformed PK parameters $\mathrm{C}_{\max }, \mathrm{AUC}_{0-\mathrm{t}}$ and $\mathrm{AUC}_{0-\infty}$ at $5 \%$ level of significance. No statistically significant difference was observed in Test/Reference ratio of $\mathrm{C}_{\max }, \mathrm{AUC}_{0-\mathrm{t}}$ and $\mathrm{AUC}_{0-\infty}$ of both species at 5 $\%$ level of significance.

Table 2: Least square mean values, ratios and $90 \%$ Cls for pharmacokinetic variables of Cetirizine formulations in humans

\begin{tabular}{|c|c|c|c|}
\hline \multirow[b]{2}{*}{ Parameter } & \multicolumn{2}{|l|}{ Treatment } & \multirow{2}{*}{$\begin{array}{l}\text { Ratio of LSM } \\
\text { T/R (90\% Cls) \%CV }\end{array}$} \\
\hline & $\begin{array}{l}\text { Cetirizine } \\
\text { Tablet (T) }\end{array}$ & $\begin{array}{l}\text { Zyrtec } \\
\text { Tablet }^{(\mathrm{R})}\end{array}$ & \\
\hline $\mathrm{AUC}_{0-\infty}(\mathrm{ng} \cdot \mathrm{h} / \mathrm{mL})$ & 2077.13 & 2080.79 & $99.82(96.84-102.90) 6.3$ \\
\hline $\mathrm{AUC}_{0-\mathrm{t}}(\mathrm{ng} \cdot \mathrm{h} / \mathrm{mL})$ & 1954.39 & 1954.63 & 99.99 (96.57-103.53) 6.0 \\
\hline $\mathrm{C}_{\max }(\mathrm{ng} / \mathrm{mL})$ & 225.30 & 225.26 & $100.02(95.43-104.84) 9.5$ \\
\hline
\end{tabular}


Table 3: Results of t-test for pharmacokinetic parameters

\begin{tabular}{|c|c|c|c|c|}
\hline Variable & Method & Variance & t -value & $P$-value ${ }^{*}>|t|$ \\
\hline Ratio_ $\mathrm{C}_{\max }$ & Pooled & Equal & -0.12 & 0.9088 \\
\hline Ratio_ $\mathrm{C}_{\max }$ & Satterthwaite & Unequal & -0.12 & 0.9069 \\
\hline Ratio_AUC ${ }_{0-t}$ & Pooled & Equal & -0.59 & 0.5606 \\
\hline Ratio_AUC ${ }_{0-t}$ & Satterthwaite & Unequal & -0.62 & 0.5429 \\
\hline Ratio_AUC ${ }_{0-\infty}$ & Pooled & Equal & -0.25 & 0.8063 \\
\hline Ratio_ $\mathrm{AUC}_{0-\infty}$ & Satterthwaite & Unequal & -0.25 & 0.8088 \\
\hline
\end{tabular}

Comparison of least square means ratios across beagle dog and human bioavailability study had showed $<2 \%$ variation in least square means ratios (T/R) of $\mathrm{C}_{\max }$ and $A U C$ in dog and human data. This indicates that least square means ratios (test/reference) dog and human are comparable to each other. Similar intra-subject variability observed for primary pharmacokinetic parameters in dog and human bioavailability studies. The intra-subject variability (\%CV) of $\mathrm{C}_{\max }$ and $\mathrm{AUC}$ are approximately $9 \%$ and $6 \%$, respectively. This exhibits that formulation variability, i.e., intra-subject variability will remain the same whether bioavailability study conducted on dog or human.

Outcome of bioequivalence study is depended upon ratio and $90 \% \mathrm{Cl}$ for primary pharmacokinetic parameter such as $\mathrm{C}_{\max }$ and AUC. Even though differences were observed in pharmacokinetic values as discussed above but there is no significant differences were observed on absolute $\mathrm{T} / \mathrm{R}$ ratio and intra-subject variability. The $90 \% \mathrm{Cl}$ for ratios were within the acceptance limits of $80-125 \%$ for cetirizine dog study as well as human study. The products were bioequivalent in both beagle dogs and humans.

\section{CONCLUSION}

The results of the preliminary investigation suggest that beagle dogs may be suitably substituted for human subjects in the bioequivalence studies of generic products with the result that study costs would be considerably lower

\section{ACKNOWLEDGEMENT}

These studies were funded by Ranbaxy Laboratories Limited, India.

\section{DECLARATION OF INTEREST}

Authors report no conflicts of interest. Authors alone are responsible for the content and writing of the paper.

\section{REFERENCES}

1. Medicins Frontieres. Untangling the web of price reductions. $10^{\text {th }}$ ed. Geneva, Switzerland: Medecins sans Frontieres (MSF) 2008.

2. Akimoto M, Nakamura FA, Nakamura M, Maki T, Yamada $K$, Suwa T, Ogata H. Release and absorption characteristics of chlorphenesin carbamate sustained-release formulations: In vitro-in vivo and in vivo dog-human correlations. Int J Pharm 1995; 117: 31-39.

3. Wu Y, Loper A, Landis E, Hettrick L, Novak L, Lynn K, Chen $C$, Thompson $K$, Higgins $R$, Batra $U$, Shelukar S, Kwei G, Storey $D$. The role of biopharmaceutics in the development of a clinical nanoparticle formulation of MK-0869: A beagle dog model predicts improved bioavailability and diminished food effect on absorption in human. Int J Pharm 2004; 285: 135-146.

4. Kevin WG, Sibtain R, Kennan M, John BM. Bioavailability of Generic Ritonavir and Lopinavir/Ritonavir Tablet Products in a Dog Model. J Pharm Sci 2010; 99(2):626-31.

5. Cook CP, Scott DW, Miller WH, Kirker JE, Cobb SM. Treatment of canine atopic dermatitis with cetirizine, a second generation antihistamine: $A$ single blinded, placebo-controlled study. Can Vet J 2004; 45: 414-417.

6. Guidelines for the Care and Housing of Dogs in Scientific Institutions. Animal Research Review Panel. Guideline no. 14 - 1. March 1999.

7. Guidelines for Bioequivalence Studies for Veterinary Medicinal Products. EMEA; July 2001.

8. FELASA recommendations for the health monitoring of breeding colonies and experimental units of cats, dogs and pigs: Report of the federation of european laboratory animal science associations (FELASA) working group on animal health. Laboratory animals 1998; 32: 1 - 17.

9. Committee for the Purpose of Control and Supervision of Experiments on Animals (CPCSEA) guidelines for laboratory animal facility. The Gazette of India; December 15, 1998.

10. Guide for the Care and Use of Laboratory Animals, $8^{\text {th }}$ edn, USA. National Research Council of National Academies.

11. Center for Drug Evaluation and Research (CDER), US FDA. Industry Guidance, Bioanalytical Method Validation. May 2001.

12. Kamba M, Seta Y, Kusai A, Ikeda M, Nishimura K. A unique dosage form to evaluate the mechanical destructive force in the gastrointestinal tract. Int $J$ 
Tiwari et al

Pharm 2000; 208: 61-70. 